\title{
The diagnostic dilemma of NMOSD/anti-MOG Antibody and Pseudotumor cerebri co-existence in a patient with recent COVID-19 infection.
}

\author{
Sundus Sardar ${ }^{1}$, Abeer Safan ${ }^{1}$, Lina Okar ${ }^{1}$, Nagham Sadik $^{1}$, and Gholam Adeli ${ }^{1}$ \\ ${ }^{1}$ Hamad Medical Corporation
}

December 19, 2020

\begin{abstract}
SARS-CoV-2 infection may present with numerous neurological manifestations. We report the case of a young obese lady, recently discharged after treatment for COVID-19 infection, who later presented with headache and blurred vision, diagnosed to have pseudotumor cerebri and concurrent bilateral optic neuritis.
\end{abstract}

The diagnostic dilemma of NMOSD/anti-MOG Antibody and Pseudotumor cerebri coexistence in a patient with recent COVID-19 infection.

Sundus Sardar ${ }^{1}$, Abeer Safan ${ }^{2}$, Lina Okar ${ }^{3}$, Nagham Sadik ${ }^{1}$, Gholam Adeli ${ }^{2}$

${ }^{1}$ Department of Internal Medicine, Hamad Medical Corporation, Doha, Qatar.

${ }^{2}$ Department of Neurology, Neurosciences Institute, Hamad Medical Corporation, Doha, Qatar.

${ }^{3}$ Department of Family Medicine, Hamad Medical Corporation, Doha, Qatar.

\section{Correspondence:}

Dr. Abeer Safan, Neurology Department, Neurosciences Institute, Hamad Medical Corporation, Doha, Qatar. Email: ASafan@hamad.qa

\begin{abstract}
:
SARS-CoV-2 infections have presented with numerous neurological manifestations, providing insight into the potential of SARS-CoV-2 virus in triggering autoantibody production and dysregulating the immune system. We report the case of a young obese lady, recently discharged after treatment for COVID-19, who later presented with headache and blurred vision, diagnosed to have pseudotumor cerebri and concurrent bilateral optic neuritis. To the best of our knowledge, this is the first case report to establish concurrent intracranial hypertension, anti-MOG syndrome/neuromyelitis optical optica syndrome disorder (NMOSD) and SARS-CoV-2 infection. The accumulative number of reports of COVID-19 patients with demyelinating manifestations such as bilateral optic neuritis with clinical and radiological features of NMOSD/Anti-MOG syndrome delineate that probable neuro-invasion is a reasonable concern that SARS-CoV-2 is an emerging neuropathogen. Further experimental models and research are warranted to better understand how the acute and chronic course of illness in the context of SARS-CoV-2 could further modulate our approach and treatment.
\end{abstract}

Key Clinical Message: 
Owing to autoantibody production and thrombophilic disorders in COVID-19, physicians must have low threshold to investigate secondary IIH and demyelinating disorders in patients with headache and decreased vision following recent COVID-19 infection.

Keywords: Coronavirus; SARS-CoV-2; COVID-19; Neurological manifestations; NMOSD; Anti-MOG syndrome; Pseudotumor cerebri.

\section{Introduction:}

The first case of COVID-19 emerged in Wuhan, China, in December 2019[1], following which the surge in COVID-19 cases reached a pandemic proportion by March, 2020. The spectrum of clinical presentations of COVID-19 include respiratory, cardiac, neurological, gastrointestinal, renal and ophthalmic involvement ${ }^{[2]}$. Recent reports have demonstrated the plausible neuro-invasive potential of SARS-CoV-2 infection ${ }^{[3]}$ and it has also been hypothesized that SARS-CoV-1 may trigger autoantibody production ${ }^{[4]}$. Several cases have also documented the ability of SARS-CoV-2 virus in dysregulation of the immune system, with COVID-19 cases presenting as Guillain-Barré syndrome ${ }^{[5]}$, Miller Filler syndrome ${ }^{[6]}$, Kawasaki syndrome ${ }^{[7]}$ and antiphospholipid antibody syndrome ${ }^{[4]}$.

\section{Case presentation:}

A 38-year-old female presented to the emergency in our hospital with history of headache and blurry vision. Her past medical history was significant for diabetes mellitus, obstructive sleep apnea, migraine, gastritis, and obesity. Her last hospital admission was two weeks ago, due to COVID-19 pneumonia. She was admitted for ten days and treated as our local guideline with azithromycin, hydroxychloroquine for 7 days, and amoxicillin-clavulanic acid. Her COVID-19 infection course was mild in nature as she only needed oxygen supplementation for 4 days. Regarding this admission, she described headache that increased gradually in severity since she was discharged, more severe in the morning, increase with straining, and awaken her from sleep and most importantly it is different from here usual migraine episodes. This headache was followed by blurry vision that worsened gradually over one week and difficulty distinguishing colors especially in the left eye as well as painful eye movements. She experienced nausea few times but no vomiting. Initial vitals were normal with temperature of $36.8{ }^{\circ} \mathrm{C}$, respiratory rate of 19 breaths per minute, blood pressure of $145 / 80 \mathrm{mmHg}$, oxygen saturation of $99 \%$, and weight of $117.3 \mathrm{~kg}$. Ophthalmology examination showed severe optic disc swelling on the left and mild on the right side, color vision $1 / 7$ in the left eye and $7 / 7$ in the right. Labs were only remarkable for microcytic anemia. COVID-19 PCR was negative on current admission. CT head revealed slightly bulky left optic nerve with optic disc swelling without CT evidence of acute intracranial abnormality. Here, the patient was admitted to the inpatient medical ward with a clinical picture of idiopathic intracranial hypertension. Lumbar puncture (LP) revealed an opening pressure of $45 \mathrm{~cm}$ $\mathrm{H}_{2} \mathrm{O}$, and $40 \mathrm{~mL}$ clear and colorless CSF was drained at this time. With evidence of increased intracranial pressure by LP findings, acetazolamide $250 \mathrm{mg}$ TID was initiated. After that, the patient reported mild improvement in her headache but her visual acuity remained the same. We decided to monitor her for few days for possible improvement, in the fourth day as her vision still deteriorated. Another LP was performed which revealed normal opening pressure of $18 \mathrm{cmH}_{2} \mathrm{O}$ with normal CSF analysis. MRI bilateral orbit (Figure 1) revealed effacement of the perioptic optic CSF space, mild diffuse increased $\mathrm{T} 2$ signal principally involving the of optic nerve entire intra-orbital segment extending anteriorly to the papilla, with mildly raised optic disc, showing significant diffusion restriction on DWI series and post contrast optic nerve and perioptic enhancement with mildly raised enhancing papilla/ optic nerve insertion.

Above described appearances are suggestive of possible acute inflammatory versus demyelinating process (NMOSD) of the optic nerve on both sides. The bilateral involvement as well as the extent of involvement are suggestive of anti-MOG optic neuritis. There were no findings suggesting ischemic optic neuropathy or increased intracranial tension.

MRI orbit results raised our suspicion that it might be COVID-related post-infectious optic neuropathy due to her history of recent COVID-19 infection. At this point we decided to start IV methylprednisolone for 5 days course and acetazolamide dose was increased to 500mg BID. Aquaporin-4 antibodies and oligoclonal 
bands were negative. Despite a 7-day course of steroids, the patient reported only mild improvement in vision. However, visual acuity regressed after completion of course of steroids. She was started on plasma exchange for further management; however, she developed anaphylactic reaction and thus, plasma exchange was aborted. Her serum IgA level assessed prior to plasma exchange was normal. She then received a trial of intravenous immunoglobulin (IVIG) for 5 days after which she reported significant improvement in her visual acuity and she was discharged home.

\section{Discussion:}

Since March 2020, the World health organization (WHO) declared the severe acute respiratory syndrome coronavirus 2 (SARS-CoV-2) infections to have reached pandemic proportions. Ever since, COVID-19 infections have rapidly spread to reach more than 63.9 million reported cases to date. The virus is well known with its portentous respiratory system manifestations, but compelling evidence from experimental study and case reports points towards its potential neurotropism ${ }^{[8][9]}$.

SARS-CoV-2 has demonstrated a wide array of manifestation and its potent ability to ignite a profound host immune response varying from ARDS seen in $29 \%$ of cases to cytokine storms that warrant immunotherapy. ${ }^{[10]}$ Multiple neurological manifestation is well recognized which could be gauged on a spectrum of severity, from anosmia, headaches to stroke syndrome, encephalitis, myelitis, and demyelination in close proximity to the illness. ${ }^{[9]}$

V. Montalvan in May 2020, published a systematic review highlighting the possible neuropathogenicity of the virus. It proposed possible invasion of cerebral circulation endothelium via hematogenous spread of SARS$\mathrm{CoV}-2$ from systemic circulation and potential viral propagation through the cribriform plate and olfactory bulb. ${ }^{[8]}$

Neuromyelitis optica spectrum disorder (NMOSD) and anti-myelin oligodendrocyte glycoprotein (anti-MOG) syndromes are two distinct conditions owing to their proposed pathophysiology and diagnostic antibodies yet have compelling and over-lapping features ${ }^{[11]}$. As both exhibit demyelinating and inflammatory immune mediated responses on to the central nervous system with predilection to involve the spinal cord and optic nerve bilaterally which makes them well differentiated from multiple sclerosis (MS ${ }^{[1]}$.

It is estimated that NMOSD holds a prevalence of 0.5 to 10 per 100,000, with well recognized ethnic and geographical discrepancies ${ }^{[12][13]}$. Like most of auto-immune conditions, NMOSD is not an exception with incidence 10 times more common in women. ${ }^{[11]}$ Despite the fact, monophasic NMOSD seems to affect both genders equally with a median age of onset is 32 to 41 years, yet cases in children and adolescent has been reported $^{[12]}$.

The hallmark of NMOSD is well recognized with its preferential involvement of optic nerve and the spinal cord as acute transverse myelitis associated with a longitudinally extensive transverse myelitis (LETM) lesion on spinal MRI ${ }^{[14][15] 16][17]}$. NMO-IgG discovery in 2004, lead to a spectrum of brainstem and diencephalic signs that are well recognized to institute a pivotal part of the 2015 diagnostic criteria [nternational Panel for NMO Diagnosis [IPND $]^{[14]}$.

Clinical analysis of the largest international cohort to date published ${ }^{[17]}$ of AQP4-seropositive NMOSD, shows myelitis was the initial manifestation in $48 \%$, optic neuritis in $42 \%$, and simultaneous optic neuritis and myelitis in $4 \%^{[14][17]}$. Interestingly enough, NMOSD-associated optic neuritis is extensive; it could manifest as severe visual loss at onset, and bilateral involvement of the optic nerves or optic chiasm. It is observed to exhibit a relapsing course with poor response to IV corticosteroid pulses, poor recovery with permanent visual deficits, with relatively unspecific lesions or normal brain MRI. [14]

Anti-MOG syndromes are defined as AQP4-seronegative patients with a phenotype of NMOSD [12][14][15]. On the contrary, they have lower female to male ratio, with earlier age of onset, when compared to sero-positive AQP4-IgG NMOSD as per major published series. ${ }^{[14]}$

In 2017, a large cross-sectional study of 132 patients with non-MS demyelinating disease, estimated that $73 \%$ 
fulfilled the diagnostic criteria of 2015 International Panel for NMO diagnosis have aquaporin-4 antibodies (AQP4-IgG), around $11 \%$ were MOG-IgG seropositive and $16 \%$ remained seronegative ${ }^{[16]}$.

Despite the overlapping features between NMOSD and anti-MOG syndrome, optic neuritis in the latter exhibits distinctive feature that could help us distinguish it from optic neuritis in AQP4-IgG NMOSD and $\mathrm{MS}^{[14][17]}$. Bilateral optic neuritis with radiographic evidence of longitudinally extensive lesions in the retrobulbar and orbital segments of the optic nerve, with one third of patients exhibiting perioptic contrast enhancement ${ }^{[14][18]}$. Chiasmal involvement is very rare in anti-MOG syndromes as opposed to NMOSD ${ }^{[18]}$.

Myelin oligodendrocyte glycoprotein constitutes $0.05 \%$ of total myelin proteins; its significance was encountered with the introduction of experimental autoimmune encephalomyelitis (EAE) as an animal model of demyelination ${ }^{[19]}$. Recent evidence suggests that anti-MOG antibodies are produced peripherally, making their way to the CNS following a breakdown in the blood-brain barrier due to infections. To support this notion, the absence of restrict oligoclonal bands in CSF of patients with anti-MOG syndromes patients supports its peripheral origin ${ }^{[20][21]}$. Molecular mimicry could explain the pathophysiology in anti-MOG antibodies production, as $50 \%$ of patient reported history of receding infectious prodrome ${ }^{[21]}$.

Since the early 1790 , an etiologic correlation between prodromal viral illness and parainfectious or postinfectious demyelinating syndromes is well recognized, with the description of a 23-year-old woman developed encephalomyelitis 1 week after a measles rash ${ }^{[22]}$. Anti MOG syndromes, Guillain-Barré syndromes and NMOSD in SARS-Cov-2 infection is well reported in a number of cases, with one for the prevailing mechanism of injury is likely to involve molecular mimicry; with various viral antigens, trigger an immune response toward endogenous CNS myelin proteins, including MOG ${ }^{[22][23]}$.

On the other hand, idiopathic intracranial hypertension (IIH) constitutes of a constellation of signs and symptoms of raised intracranial pressure with fulfillment of Dandy's criteria. IIH has an annual incidence of 19.3 per 100,000 in those who weigh $20 \%$ or more than their ideal body weight, with $90 \%$ affecting females ${ }^{[24]}$. Different pathophysiology varying from vascular, hormonal, and increased CSF outflow resistance have been proposed, which could be directly linked to common risk factors ${ }^{[24]}$.

Recent evidence has linked interleukin-6 (IL-6) to disease activity in NMOSD by mechanism of promoting survival of plasmablasts, stimulation of AQP4-IgG secretion, altering integrity and functionality of BBB and increasing differentiation and activation of pro-inflammatory T-lymphocytes. In patients with NMOSD, IL-6 levels in the serum and CSF are significantly increased [25]. Interlinking this to elevated IL-6 levels in SARS-CoV-2 infection may explain the underlying pathophysiology for occurrence of NMOSD in cases of recent COVID-19 infection.

Our patient presented with a severe progressive headache after recent COVID-19 infection, followed by rapid deterioration in her vision, with severe optic disc edema and normal MRI head. Lumbar puncture (LP) showed high opening pressure $(45 \mathrm{cmH} 2 \mathrm{O})$ and normal CSF analysis, with headache that improved with therapeutic LP and acetazolamide. MRI orbits was pursued due to further deterioration of her vision, which showed bilateral increased T2 signal involving optic nerve entire intra-orbital segment extending anteriorly to the papilla with post-contrast optic nerve and perioptic enhancement, thereby raising the suspicion of optic neuritis due to anti-MOG syndrome or NMOSD in correlation with SARS-CoV-2. With multiple sessions of IVIG, her visual acuity improved.

\section{Conclusion:}

COVID-19-associated neurological manifestations are widely reported, with some rare instances of anti-MOG syndrome in coexistence with SARS-CoV-2 infection. We report the first case of concurrent NMOSD and IIH in the context of COVID-19 infection. While the propensity for triggering autoantibody production and thrombophilic disorders is prevalent in COVID-19, physicians and neurologists must be vigilant with low threshold to further investigate the possibility of secondary idiopathic intracranial hypertension and demyelinating disorders in patients presenting with headache and decreased visual acuity following recent COVID-19 infection. 


\section{Images:}

Figure 1: MRI orbit showing effacement of the perioptic-optic CSF space, mild diffuse increase in T2 signal involving the optic nerve, entire intraorbital segment extending anteriorly to the papilla, with significant diffusion restriction on DWI series and post-contrast optic nerve and peri-optic enhancement.

\section{Acknowledgements}

None.

\section{Consent}

Written informed consent was obtained from the patient for publication of this case report. A copy of the written consent is available for review by the Editor-in-Chief of this journal.

\section{Conflicts of Interest}

The authors have no conflict of interest to disclose.

\section{Funding}

Qatar National Library funded the open access publication fees of this case.

\section{Author contributions}

Writing the initial draft of the manuscript: Sundus Sardar, Abeer Safan, Lina Okar

Conceptualization and supervision: Naghem Sadik, Gholam Adeli

Medical management of the case: Sundus Sardar, Abeer Safan, Naghem Sadik, Gholam Adeli

Revising the manuscript critically and literature review: Sundus Sardar, Abeer Safan, Lina Okar, Naghem Sadik, Gholam Adeli

The first authors (SS and AS) contributed equally to the writing and preparation of this article. SS, AS, and LO have written the initial draft of the manuscript and performed the literature review. The draft was revised and updated by SS, AS, LO with supervision from NS and GA. SS, AS, NS, GA were part of the medical treating team. All the authors critically reviewed the initial and the final draft of the manuscript and approved it for submission.

\section{Appendix 1}

\begin{tabular}{lll}
\hline Name & Location & Contribution \\
\hline Sundus Sardar & Internal Medicine, Hamad Medical Corporation, Doha, Qatar & Writing the initial draft of the manu \\
Abeer Safan & Neurosciences Institute, Hamad Medical Corporation, Doha, Qatar & Writing the initial draft of the manu \\
Lina Okar & Family Medicine, Hamad Medical Corporation, Doha, Qatar & Writing the initial draft, revising the \\
Nagham Sadik & Internal Medicine, Hamad Medical Corporation, Doha, Qatar & Conceptualization and supervision, \\
Gholam Adeli & Neurosciences Institute, Hamad Medical Corporation, Doha, Qatar & Conceptualization and supervision, r \\
\hline
\end{tabular}

\section{References:}

[1] Wu F, Zhao S, Yu B, Chen YM, et al. A new coronavirus associated with human respiratory disease in China. Nature. 2020;579:265-269.

[2] Gupta, A., Madhavan, M.V., Sehgal, K. et al. Extrapulmonary manifestations of COVID-19. Nat Med 26, 1017-1032 (2020). https://doi.org/10.1038/s41591-020-0968-3

[3] Li YC, Bai WZ, Hashikawa T. The neuroinvasive potential of SARS-CoV2 may play a role in the respiratory failure of COVID-19 patients. J Med Virol. 2020;92:552-555. 
[4] Zhang Y, Xiao M, Zhang S, et al. Coagulopathy and antiphospholipid antibodies in patients with covid19. N Engl J Med. 2020.

[5] Toscano G, Palmerini F, Ravaglia S, Ruiz L, Invernizzi P, Cuzzoni MG, Franciotta D, Baldanti F, Daturi R, Postorino P, Cavallini A, Micieli G. Guillain-Barré syndrome associated with SARS-CoV-2. New Engl J Med. 2020;382:2574-2576.

[6] Gutiérrez-Ortiz C, Méndez A, Rodrigo-Rey S, San Pedro-Murillo E, Bermejo-Guerrero L, Gordo-Mañas R, de Aragón-Gómez F, Benito-León J. Miller Fisher Syndrome and polyneuritis cranialis in COVID-19. Neurology 2020. doi: 10.1212/WNL.0000000000009619.

[7] Jones VG, Mills M, Suarez D, Hogan CA, Yeh D, Bradley Segal J, Nguyen EL, Barsh GR, Maskatia S, Mathew R. COVID-19 and Kawasaki disease: novel virus and novel case. Hosp Pediatr. 2020;10:537-540.

[8] Montalvan V, Lee J, Bueso T, De Toledo J, Rivas K. Neurological manifestations of COVID19 and other coronavirus infections: A systematic review. Clin Neurol Neurosurg . 2020;194:105921. doi:10.1016/j.clineuro.2020.105921

[9] Wang D, Hu B, Hu C, et al. Clinical Characteristics of 138 Hospitalized Patients With 2019 Novel Coronavirus-Infected Pneumonia in Wuhan, China [published online ahead of print, 2020 Feb 7]. JAMA . 2020;323(11):1061-1069. doi:10.1001/jama.2020.1585

[10] Croft PB. Para-infectious and post-vaccinal encephalomyelitis. Postgrad Med J . 1969;45(524):392-400. doi:10.1136/pgmj.45.524.392

[11] Lana-Peixoto MA, Talim N. Neuromyelitis Optica Spectrum Disorder and Anti-MOG Syndromes. Biomedicines . 2019;7(2):42. Published 2019 Jun 12. doi:10.3390/biomedicines7020042

[12] Mealy MA, Wingerchuk DM, Greenberg BM, Levy M. Epidemiology of neuromyelitis optica in the United States: a multicenter analysis. Arch Neurol . 2012;69(9):1176-1180. doi:10.1001/archneurol.2012.314

[13] Mori M, Kuwabara S, Paul F. Worldwide prevalence of neuromyelitis optica spectrum disorders. J Neurol Neurosurg Psychiatry . 2018;89(6):555-556. doi:10.1136/jnnp-2017-317566

[14] Jurynczyk M., Tackley G., Kong Y., Geraldes R., Matthews L., Woodhall M., Waters P., Kuker W., Craner M., Weir A., et al. Brain lesion distribution criteria distinguish ms from aqp4-antibody nmosd and mog-antibody disease. J. Neurol. Neurosurg. Psychiatry. 2017;88:132-136. doi: 10.1136/jnnp-2016-314005.

[15] Merabtene L, Vignal Clermont C, Deschamps R. Neuropathie optique dans le syndrome des anticorps anti MOG positif [Optic neuropathy in positive anti-MOG antibody syndrome]. J Fr Ophtalmol . 2019;42(10):1100-1110. doi:10.1016/j.jfo.2019.06.006

[16] Hamid SHM, Whittam D, Mutch K, et al. What proportion of AQP4-IgG-negative NMO spectrum disorder patients are MOG-IgG positive? A cross sectional study of 132 patients. J Neurol . 2017;264(10):20882094. doi:10.1007/s00415-017-8596-7

[17] Kim SH, Mealy MA, Levy M, et al. Racial differences in neuromyelitis optica spectrum disorder. Neurology . 2018;91(22):e2089-e2099. doi:10.1212/WNL.0000000000006574

[18] Jarius S, Ruprecht K, Kleiter I, et al. MOG-IgG in NMO and related disorders: a multicenter study of 50 patients. Part 2: Epidemiology, clinical presentation, radiological and laboratory features, treatment responses, and long-term outcome. J Neuroinflammation . 2016;13(1):280. Published 2016 Sep 27. doi:10.1186/s12974-016-0718-0

[19] Berger T, Rubner P, Schautzer F, et al. Antimyelin antibodies as a predictor of clinically definite multiple sclerosis after a first demyelinating event. $N$ Engl J Med . 2003;349(2):139-145. doi:10.1056/NEJMoa022328

[20] Ramanathan S, Mohammad S, Tantsis E, et al. Clinical course, therapeutic responses and outcomes in relapsing MOG antibody-associated demyelination. J Neurol Neurosurg Psychiatry . 2018;89(2):127-137. 
doi:10.1136/jnnp-2017-316880

[21] Reindl M, Di Pauli F, Rostásy K, Berger T. The spectrum of MOG autoantibody-associated demyelinating diseases. Nat Rev Neurol . 2013;9(8):455-461. doi:10.1038/nrneurol.2013.118

[22] Croft PB. Para-infectious and post-vaccinal encephalomyelitis. Postgrad Med J . 1969;45(524):392-400. doi:10.1136/pgmj.45.524.392

[23] Zhou S, Jones-Lopez EC, Soneji DJ, Azevedo CJ, Patel VR. Myelin Oligodendrocyte Glycoprotein Antibody-Associated Optic Neuritis and Myelitis in COVID-19. J Neuroophthalmol . 2020;40(3):398-402. doi:10.1097/WNO.0000000000001049

[24] Mondragon J, Klovenski V. Pseudotumor Cerebri. [Updated 2020 Jul 10]. In: StatPearls [Internet]. Treasure Island (FL): StatPearls Publishing; 2020 Jan-. Available from: https://www.ncbi.nlm.nih.gov/books/NBK536924/

[25] Fujihara K, Bennett JL, de Seze J, Haramura M, Kleiter I, Weinshenker BG, Kang D, Mughal T, Yamamura T. Interleukin-6 in neuromyelitis optica spectrum disorder pathophysiology. NeurologyNeuroimmunology Neuroinflammation. 2020 Sep 3;7(5).

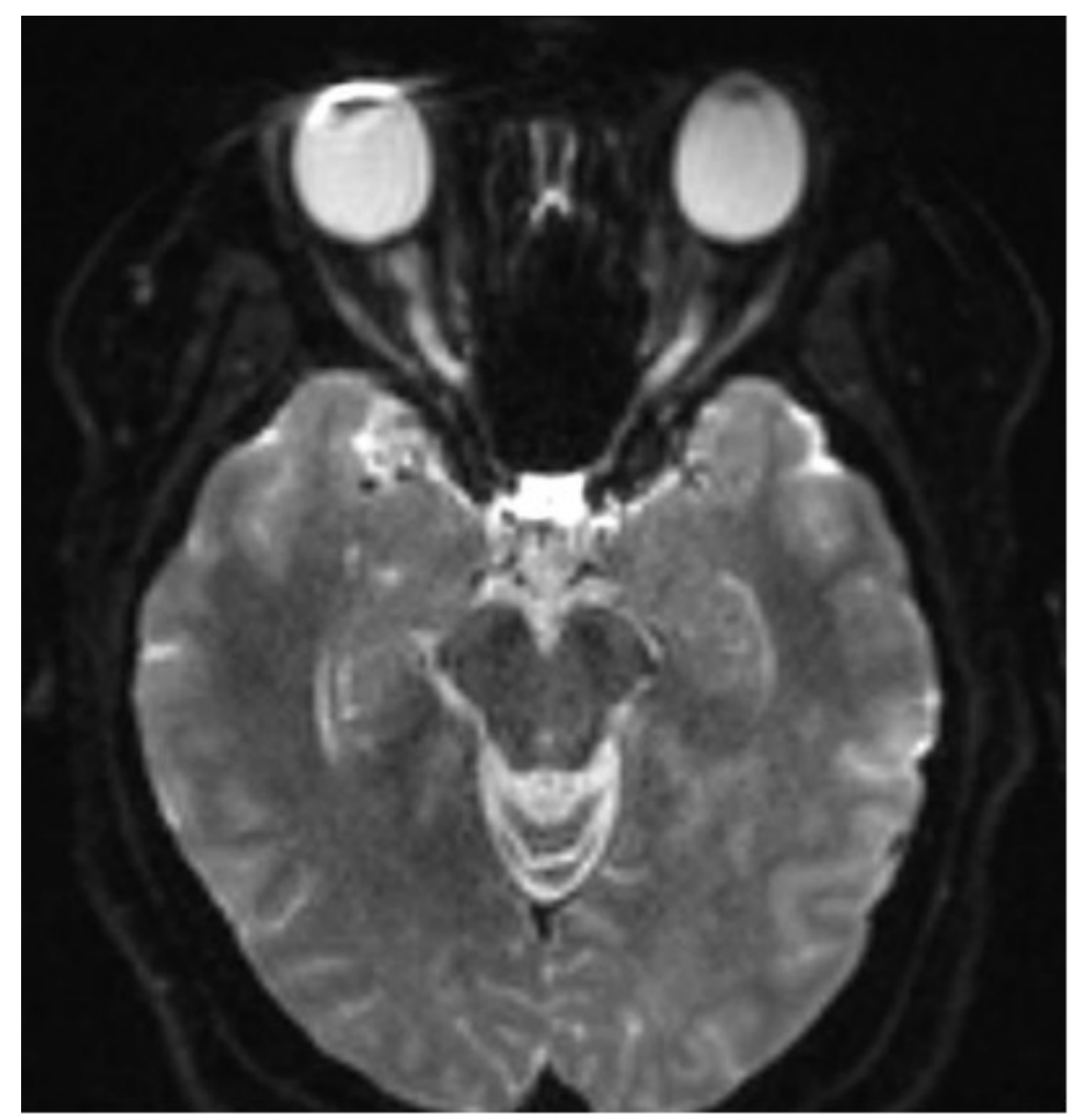

